Inflammatory bowel disease

\section{Transglutaminases: new target molecules for inflammatory bowel disease?}

\section{B Siegmund, M Zeitz}

Transglutaminases may be involved in intestinal inflammation and may even represent therapeutic targets for ulcerative colitis

$\mathrm{T}$ he family of transglutaminases (TG) includes the plasma form factor XIIIa as well as the tissue transglutaminase (tTG) and keratinocyte transglutaminase (TGk). In particular, tTG reminds every gastroenterologist primarily of coeliac disease where tTG represents the key autoantigen. ${ }^{1}$ Disease induction is confined to tTG, a ubiquitous enzyme which is released from fibroblasts, endothelial, and inflammatory cells during mechanical irritation or inflammation. At acidic $\mathrm{pH}$, which occurs with inflammation, tTG can, apart from its physiological function described in more detail below, also simply deamidate some of the glutamine residues of the gluten peptides. In coeliac disease, deamidation introduces a negative charge into the gluten peptides which can increase the binding affinity to HLA-DQ2 or HLA-DQ8, the primary HLA association in coeliac disease. Binding of gluten peptide to either HLA-DQ2 or HLA-DQ8 results in an increase in their capacity to stimulate $\mathrm{T}$ cells, thus inducing intestinal inflammation. ${ }^{23}$

As indicated in this issue of Gut by $\mathrm{D}^{\prime}$ Argenio and colleagues, ${ }^{4} \mathrm{TG}$ may be involved in intestinal inflammation in a completely different way and may even represent a therapeutic target (see page 496). But how can the physiological function of TG be defined? TG are enzymes catalysing the covalent cross linking between proteins by forming $\epsilon(\gamma$-glutamyl $)$-lysine isopeptide bonds. These bonds are formed between glutaminase and lysine residues thus stabilising intra- and extracellular proteins, a process which is required for a variety of essential physiological purposes such as barrier function in epithelia, apoptosis, and extracellular matrix formation. ${ }^{5}$ Substrates include intracellular (actin cytoskeleton, histones) as well as extracellular (collagen, vitronectin, fibronectin) proteins many of which are found in the matrix of the colonic mucosa. The balance of cross linking alters the susceptibility of the matrix to proteoly- sis and thus affects the rate of its formation and remodelling during mucosal damage and repair.

In the work presented in this issue of Gut, D'Argenio and colleagues ${ }^{4}$ hypothesise that due to impaired healing in ulcerative colitis, an imbalance of TG may participate in the inflammatory process. To approach this question, peripheral blood as well as colonic biopsies from patients with active and inactive ulcerative colitis as well as noninflamed control samples were examined for the three members of the TG family, tTG, factor XIIIa, and TGk. Endoscopic and histological disease indices were applied to determine disease activity. Factor XIIIa activity was evaluated in plasma. In addition, tTG, TGk, and factor XIIIa protein as well as RNA content in colonic tissue was analysed by western blot and reverse transcription-polymerase chain reaction, respectively. An elegant tool in this study proved to be the immunohistochemical studies visualising colonic localisation of $\mathrm{TG}$ and their reaction products, the $\epsilon(\gamma$-glutamyl $)$-lysine isopeptide bonds. With the results obtained, a scheme can be suggested as to how TG act in active ulcerative colitis. Primarily, factor XIIIa activity in plasma is significantly reduced in active disease compared with inactive ulcerative colitis or healthy controls. Immunohistochemical analysis indicated that TTG and factor XIIIa colocalise in damaged areas with the isopeptide bonds, which may explain the decrease in factor XIIIa in plasma. The TGk protein as well as RNA expression is significantly reduced in active disease in colonic tissue. tTG RNA is upregulated while tTG protein expression remains unaltered. Interestingly, in active disease, tTG appeared with two bands in the western blot analysis representing degradation probably due to endogenous proteolysis by calpain, an enzyme that inactivates tTG, known to be increased in active ulcerative colitis. ${ }^{6}$ Importantly, TGk was detected for the first time in colonic tissue where it was localised at the upper part of the crypts and it was shown that TGk was significantly downregulated in active disease. Based on these results the authors conclude that the abnormal pattern of TG contributes to the course of ulcerative colitis.

Whether or not these changes are specific for ulcerative colitis or whether they also occur in other intestinal inflammations, for example, infectious colitis, is unknown. Similar data have been obtained for Crohn's disease. ${ }^{78}$ Here, in active disease, factor XIIIa was equally decreased in serum, and immunhistochemical staining also showed colocalisation of factor XIIIa and tTG to the extracellular matrix. Interestingly, the western blot from colonic tissue from patients with active Crohn's disease showed an identical double band in active disease, an observation that was not discussed further by the authors. These data indicate that independent of the type of chronic inflammatory bowel disease, a similar pattern of TG changes can be seen. These data as well as data from the literature indicate a protective role of TG in Crohn's disease referred to above. This protective function is already evident given the functional properties of TG: TGk is abundantly expressed in epithelial cells cross linking a series of defined structural proteins, thus exerting barrier function. Consequently, the decrease in TGk is associated with a barrier defect, a well described phenomenon in intestinal inflammation. tTG serves as $G$ protein in most mammalian cells; when $\mathrm{Ca}^{2+}$ levels rise this protein becomes active in cross linking TG reactions. The potential protective effect is supported by the observation that inhibition of calpain, which can inactivate tTG, reduced colonic injury in experimental colitis. ${ }^{2}$ Thus wound healing or tissue remodelling is an active process consisting of a destructive part, including extracellular proteinases such as metalloproteinases or calpain, and a constructive part involving processing enzymes such as TG. During acute inflammation the presence of extracellular matrix proteins increases, thus inactivating at least part of the $\mathrm{TG}$, as indicated in the present study as well as in the study referred to above in Crohn's disease patients. ${ }^{9}$ Additional experimental data support this theory. Na-butyrate enemas have been demonstrated to ameliorate experimental colitis. Nabutyrate is known to upregulate tTG expression. ${ }^{10}$ Furthermore, a case report including three patients demonstrated a beneficial effect for recombinant factor XIII concentrate in patients with inflammatory bowel disease. ${ }^{11}$ These 
results could not be confirmed in a prospective, double blind, placebo controlled study in steroid refractory patients with ulcerative colitis. A total of 28 patients were included in this study and were treated with either intravenous application of factor XIII concentrates or placebo. No beneficial effect of additional factor XIII treatment was demonstrated. ${ }^{12}$

In contrast, a proinflammatory role for TG was demonstrated in an animal model of allergic conjunctivitis. ${ }^{13}$ More precisely, dual inhibition of phospholipase $\mathrm{A}_{2} \quad\left(\mathrm{PLA}_{2}\right)$ and $\mathrm{TG}$ resulted in dramatic anti-inflammatory activity. The PLA ${ }_{2}$ enzyme family comprises cell bound as well as secretory isoforms which play a key role in arachidonic acid release during acute inflammation. ${ }^{14}$ TG catalysed post-translational modifications activate secretory $\mathrm{PLA}_{2}$, thus potentially increasing eicosanoid production during acute inflammation. ${ }^{14}$ Are these data contrary to the results obtained in the present study or are there other explanations? With regard to the data obtained in Crohn's disease patients, one could argue that this is a T helper cell type 1 (Thl) disease while the allergic model represents a classic $\mathrm{T}$ helper cell type 2 (Th2) dependent model. ${ }^{15}$ However, this explanation has to be disregarded as ulcerative colitis is considered a Th2 dependent disease, with interleukin 13 representing the key inflammatory mediator. ${ }^{16}$ Another anti-inflammatory pharmacological group, the non-steroidal anti-inflammatory agents, also significantly inhibit the release of arachidonic acid. Simultaneously, non-steroidal anti-inflammatory agents have been associated with a deteriorating effect in inflammatory bowel disease. ${ }^{17}$ One might hypothesise that in the intestinal mucosa TG are mandatory for the constructive mucosal healing process, thus the anti-inflammatory properties are superior, and eventually activation of $\mathrm{PLA}_{2}$ may even be partially anti-inflammatory in this setting. In addition, bactericidal activity in the intestine has been described for $\mathrm{PLA}_{2}$ which contributes to epithelial barrier function. ${ }^{18}$

In summary, D'Argenio and colleagues ${ }^{4}$ have provided important information for an old concept. In light of the new data, one has to consider whether the highly dynamic process modulated by TG can be used to our advantage for therapeutic interventions.

\section{ACKNOWLEDGEMENTS}

The authors wish to thank the Deutsche Forschungsgemeinschaft and the Broad Medical Research Program of the Eli and Edythe L Broad Foundation for their continued support of our research.

Gut 2005;54:443-444.

doi: $10.1136 /$ gut.2004.053223

\section{Authors' affiliations}

B Siegmund, M Zeitz, Charité

Universitätsmedizin Berlin, Campus Benjamin

Franklin, Medizinische Klinik I, Berlin,

Germany

Correspondence to: $\operatorname{Dr} B$ Siegmund, Charité Universitätsmedizin Berlin, Campus Benjamin Franklin, Medizinische Klinik I,

Hindenburgdamm 30, 12200 Berlin, Germany; britta.siegmund@charite.de

Conflict of interest: None declared.

\section{REFERENCES}

1 Sollid LM. Coeliac disease: dissecting a complex inflammatory disorder. Nat Rev Immunol 2002; 2:647-55.

2 Ciclitira PJ, King AL, Fraser JS, et al. AGA technical review on celiac sprue. American Gastroenterological Association.

Gastroenterology 2001;120:1526-40.
3 Schuppan D, Dieterich W, Ehnis T, et al. Current concepts of celiac disease pathogenesis. Gastroenterology 2000;119:234-42.

4 D'Argenio G, Calvani M, Della Valle N, et al. Differential expression of multiple transglutaminases in human colon: impaired keratinocyte transglutaminase expression in ulcerative colitis. Gut 2005;54:496-502.

5 Lorand L, Graham RM, Boirivant M, et al. Transglutaminases: crosslinking enzymes with pleiotropic functions. Nat Rev Mol Cell Biol 2003;4:140-56.

6 Ballestar E, Abad C, Franco L. Core histones are glutaminyl substrates for tissue transglutaminase. J Biol Chem 1996;271:18817-24.

7 Chamouard P, Grunebaum L, Wiesel ML, et al. Significance of diminished factor XIII in Crohn's disease. Am J Gastroenterol 1998;93:610-24.

8 D'Argenio G, Biancone L, Cosenza V, et al. Transglutaminases in Crohn's disease. Gut 1995;37:690-5.

9 D'Argenio G, lovino P, Cosenza V, et al. Transglutaminase in azoxymethane-induced colon cancer in the rat. Dig Dis Sci 1995;40:685-95.

10 Scheppach W, Sommer H, Kirchner T, et al. Effect of butyrate enemas on the colonic mucosa in distal ulcerative colitis. Gastroenterology 1992; 103:51-6.

11 Lorenz R, Heinmuller $M$, Classen $M$, et al. Substitution of factor XIII: a therapeutic approach to ulcerative colitis. Haemostasis 1991;21:5-9.

12 Bregenzer N, Caesar I, Andus T, et al. Lack of clinical efficacy of additional factor XIII treatment in patients with steroid refractory colitis. The Factor XIII Study Group. Z Gastroenterol 1999:37:999-1004.

13 Sohn J, Kim TI, Yoon YH, et al. Novel transglutaminase inhibitors reverse the inflammation of allergic conjunctivitis. J Clin Invest 2003;111:121-8.

14 Kudo I, Murakami M. Phospholipase A2 enzymes. Prostaglandins Other Lipid Mediat 2002;68-69:3-58

15 Neurath MF, Finotto S, Fuss I, et al. Regulation of T-cell apoptosis in inflammatory bowel disease: to die or not to die, that is the mucosal question. Trends Immunol $2001 ; 22: 21-6$.

16 Fuss IJ, Heller F, Boirivant M, et al. Nonclassical CDId-restricted NK T cells that produce IL-13 characterize an atypical Th2 response in ulcerative colitis. J Clin Invest 2004; 113:1490-7

17 Kaufmann HJ, Taubin HL, D'Argenio G, et al. Nonsteroidal anti-inflammatory drugs activate quiescent inflammatory bowel disease. Ann Intern Med 1987; 107:513-16.

18 Harwig SS, Tan L, Qu XD, et al. Bactericidal properties of murine intestinal phospholipase A2. J Clin Invest 1995;95:603-10.

instances, is a heterodimer composed of a p50 and p65 subunit. In most mammalian cells $\mathrm{NF \kappa B}$ is found in the resting state in the cytoplasm where it is bound in a complex to a protein that is a member of a family of specific inhibitors (IKK). Following phosphorylation, the inhibitor is rapidly degraded and the released $\mathrm{NF \kappa B}$ migrates within minutes into the nucleus where it can specifically induce gene expression by binding to sequence defined DNA elements in gene promoter regions.

Even after more than a decade of mechanistic and clinical studies, the role of NFKB in intestinal inflammation is not fully understood as its activity as a transcription factor is rather promiscuous. While activation of $N F \kappa B$ is a strong inducer of expression of proinflammatory molecules, the sequences
$\mathrm{N}$ uclear factor kappa B (NFкB) was discovered as a transcription factor some 15 years ago. ${ }^{1}$ Since then the protein has been linked to early pathophysiological events in a host of inflammatory conditions. NF $\kappa \mathrm{B}$, in most 
of many genes encoding for proteins involved in contrainflammatory regulation also have NFKB binding sites. In addition, dimers with a different composition (that is, p50-p50 homodimers) appear to exist as endogenous inhibitors of NFKB (p65) induced gene activation. ${ }^{2}$

In Crohn's disease, mechanistic data have been generated in an animal model that undoubtedly demonstrated that activation of NFKB is intimately linked to mucosal inflammation and destruction. $^{3}$ The use of antisense constructs to silence NFкB (p65) greatly improved inflammatory lesions in the murine interleukin 10 knockout model of inflammatory bowel disease (IBD) and also resolved inflammatory events in human intestinal cells from patients with IBD. ${ }^{3}$ Therefore, NFKB (p65) was thought to be one of the main drivers of the inflammatory reaction. This mechanistic proof was supported by descriptive studies reporting high levels of NFKB (p65) activation in the intestinal mucosa of patients with IBD. ${ }^{45}$ Activation of NFKB therefore appeared to be a hallmark of the pathophysiology of IBD. Clinical development of the use of antisense constructs to silence $\mathrm{NF \kappa B}$ (p65) has therefore begun.

The first question on the true role of $\mathrm{NF \kappa B}$ in the pathophysiology of IBD arose when the genetic aetiology of Crohn's disease was unveiled. The discovery of NOD2 (CARD15) as a disease gene demonstrated a series of genetic defects that led to a deficit in NFкB activation in various cell types in response to bacterial stimulation. ${ }^{6-8}$ This apparent contradiction was resolved through a pathophysiological model in which mucosal barrier function (that is, maintained by "controlled" NFкB activation) was impaired by genetic variants in the NOD2 gene. ${ }^{9}$ The defect in NOD2 mediated NFKB activation in response to bacterial stimulation then leads to compromised host defence which allows the mucosal barrier to be over run with non-pathological "normal" bowel flora. This model allows for uncontrolled widespread activation of $\mathrm{NF \kappa B}$ as the final consequence of a defective innate immune barrier. As a possible non-genetic mechanism to trigger disease manifestation, simplification of the flora was suggested that would lead to a higher invasive pressure of the remaining reduced number of normal bacterial species. ${ }^{10}$

We are now confronted with yet another finding that is difficult to integrate into the current model of pathophysiology. In this issue of Gut, Andresen and colleagues ${ }^{11}$ describe high levels of NFKB activation in collagenous colitis that are indistinguishable by means of immunohistology and cell biology from the pattern of $\mathrm{NF \kappa B}$ activation found in ulcerative colitis (see page 503). The finding is supported by similar patterns of IKK deactivation and increased activation of NFKB target genes, respectively. However, collagenous colitis and ulcerative colitis are distinctly different diseases with regard to their microscopic and macroscopic pathology. Hence the findings of Andresen and colleagues ${ }^{11}$ would imply

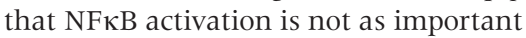
as previously believed in the pathophysiology of the destructive mucosal inflammatory reaction in IBD and could therefore be an epiphenomenon. If future work supports this notion, our understanding of the role of NFкB activation in the pathophysiology of intestinal inflammation may be challenged. At present it is difficult to seamlessly integrate these new findings into a unifying hypothetical model. Further work on the spatial and cellular distribution of NFKB activation in the mucosa of IBD patients and in other inflammatory conditions is necessary. Clinical results of ongoing therapeutic studies in which NFאB (p65) is specifically silenced by rectal topical application of an antisense construct may be a pivotal step forward in our understanding of the role of $\mathrm{NF \kappa B}$ in the pathophysiology of human IBD.

Gut 2005;54:444-445.

doi: $10.1136 /$ gut.2004.051797

Correspondence to: Professor S Schreiber, Hospital for General Internal Medicine and Institute for Clinical Molecular Biology, Christian-Albrechts-University,

Schittenhelmstrasse 12, 24105 Kiel, Germany; s.schreiber@mucosa.de

Conflict of interest: None declared.

\section{REFERENCES}

1 Barnes PJ, Karin M. Nuclear factor-kappaB: a pivotal transcription factor in chronic inflammatory diseases. N Engl J Med 1997;336: 1066-71.

2 Ziegler-Heitbrock HW, Wedel A, Schraut W, et al. Tolerance to lipopolysaccharide involves mobilization of nuclear factor kappa $B$ with predominance of p50 homodimers. J Biol Chem 1994;269:17001-4.

3 Neurath MF, Pettersson S, Meyer zum Buschenfelde $\mathrm{KH}$, et al. Local administration of antisense phosphorothioate oligonucleotides to the p65 subunit of NF-kappa B abrogates established experimental colitis in mice. Nat Med 1996;2:998-1004.

4 Schreiber S, Nikolaus S, Hampe J. Activation of nuclear factor kappa $B$ in inflammatory bowel disease. Gut 1998;42:477-84.

5 Rogler G, Brand K, Vogl D, et al. Nuclear factor kappaB is activated in macrophages and epithelial cells of inflamed intestinal mucosa. Gastroenterology 1998; 115:357-69.

6 Hugot JP, Chamaillard $M$, Zouali $\mathrm{H}$, et al. Association of NOD2 leucine-rich repeat variants with susceptibility to Crohn's disease. Nature 2001;411:599-603.

7 Ogura Y, Bonen DK, Inohara N, et al. A frameshift mutation in NOD2 associated with susceptibility to Crohn's disease. Nature 2001;411:603-6.

8 Hampe J, Cuthbert A, Croucher PJ, et al. Association between insertion mutation in NOD2 gene and Crohn's disease in German and British populations. Lancet 2001;357:1925-8.

9 Ogura Y, Inohara N, Benito A, et al. Nod2, a Nod1/Apaf-1 family member that is restricted to monocytes and activates NF-kappaB. J Biol Chem 2001;276:4812-18

10 Ott SJ, Musfeldt M, Wenderoth DF, et al. Reduction in diversity of the colonic mucosa associated bacterial microflora in patients with active inflammatory bowel disease. Gut 2004; 53:685-93.

11 Andresen L, Jørgensen VL, Perner A, et al. Activation of nuclear factor $\kappa B$ in colonic mucosa from patients with collagenous and ulcerative colitis. Gut 2005;54:503-9. 


\section{Where there's smoke there's not necessarily fire}

\section{V Apte, R C Pirola, J S Wilson}

\section{The role of smoking in alcoholic pancreatitis}

$\Lambda$ though alcohol abuse is a major association of chronic pancreatitis, it is well known that only a minority of heavy drinkers develop clinically evident pancreatitis. ${ }^{12}$ This observation has led to a sustained effort to identify factors that may increase the susceptibility of alcoholics to the development and progression of the disease.

One of the candidate susceptibility factors is smoking. The interest in smoking as a risk factor for the development and accelerated progression of alcoholic pancreatitis is understandable given that a number of smoking/nicotine related effects on the pancreas have been described in the literature. High concentrations of nicotine have been shown to increase pancreatic protein synthesis in isolated acini. ${ }^{3}$ Nicotine has also been shown to induce vacuolisation and nuclear pyknosis in acinar cells. ${ }^{4}$ Serum levels of pancreatic enzymes are reported to be significantly increased in smokers after intravenous secretin..$^{5-7}$ In addition, in vivo and in vitro studies have demonstrated that smoking significantly inhibits pancreatic secretion. $^{89}$ The concept that smoking enhances the toxic effects of alcohol on the pancreas was examined in a recent experimental study where cigarette smoke was administered to anaesthetised rats receiving intravenous ethanol; the authors reported that alcohol induced pancreatic ischaemia in rats was significantly potentiated by exposure to cigarette smoke. ${ }^{10}$

In this issue of Gut, Maisonneuve and colleagues ${ }^{11}$ have investigated the potential role of smoking in alcoholic pancreatitis using a large cohort $(n=934)$ of patients with chronic alcoholic pancreatitis (see page 510). The protocol consisted of a retrospective examination of data retrieved from clinical records or computerised databases. Demographic and clinical characteristics were noted as were the date of diagnosis, smoking status, alcohol consumption, and the presence of calcification and/or diabetes at diagnosis. The authors reported that smokers were diagnosed with pancreatitis at a younger age than non-smokers and that the prevalence of calcification at diagnosis was significantly higher in the smoking group. The authors further noted that smoking was significantly associated with the development of calcification after the initial diagnosis of pancreatitis; however, this appeared to be independent of the "smoking

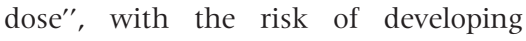
calcifications being similar in moderate smokers $(<1$ pack/day) and heavy smokers ( $>1$ pack/day).

The observation by Maisonneuve and colleagues $^{11}$ that alcoholic pancreatitis develops earlier in smokers than in nonsmokers is similar to that reported previously by Bourliere and colleagues. ${ }^{12}$ However, both of these studies have been confounded by the fact that compared with non-smokers, a higher proportion of smokers were also heavy drinkers. Thus the groups being compared were potentially different with respect to two variables, alcohol intake and tobacco consumption. It is notable that when the amount of alcohol consumed in the two groups was controlled for, as in the study of Haber et al in $1993,{ }^{13}$ no association was detected between smoking and pancreatitis. This finding highlights a point of critical importance for any studies examining individual susceptibility to alcoholic pancreatitis, that the essential comparison for such studies must be between alcoholics with the disease and alcoholics without the disease so that the index and the control groups differ in only one variable (that is, the presence or absence of pancreatitis). Over the past two decades, a number of candidate susceptibility factors have been examined, including diet, amount and type of alcohol consumed, pattern of alcohol consumption, lipid intolerance (see review by Haber and colleagues ${ }^{14}$ ) and smoking. ${ }^{12-15}$ Inherited factors have also been examined, including blood group antigens, HLA serotypes, ${ }^{16} \alpha_{1}$ antitrypsin phenotypes, ${ }^{17}$ cystic fibrosis genotype, ${ }^{18}$ tumour necrosis factor $\alpha$ genotype, $^{19}$ genotypes of alcohol metabolising enzymes (alcohol dehydrogenase $(\mathrm{ADH})$, aldehyde dehydrogenase (ALDH), cytochrome P4502El $\left.(\mathrm{CYP} 2 \mathrm{E} 1)^{20}\right)$, and mutations of genes related to pancreatic proteins that may play an important role in autodigestive injury to the gland (these include digestive enzymes ${ }^{21-23}$ and proteins that can inactivate digestive enzymes such as pancreatic secretory trypsin inhibitor (PSTI)). ${ }^{24} 25$ Not all of the above studies have included alcoholics without pancreatitis as controls and despite the extensive search, a predisposing factor(s) to alcoholic pancreatitis remains to be unequivocally identified.

Maisonneuve and colleagues ${ }^{11}$ are to be commended for their attempts to address an important question with respect to the pathobiology of alcoholic pancreatitis. The strength of the study lies in the relatively large cohort of subjects involved. However, in addition to the lack of appropriate controls (alcoholics without pancreatitis), the study has a number of limitations (some of which have been acknowledged by the authors themselves) and the findings need to be interpreted with some caution. The retrospective nature of data collection (relying on patient records alone) impacts significantly on the ability to accurately assess alcohol intake or cigarette smoke exposure. In the absence of accurate estimations, the classification of low, moderate, heavy, and very heavy alcohol consumption is somewhat arbitrary. Furthermore, many would argue that those patients consuming less than five drinks per day do not have alcoholic pancreatitis and that alcohol consumption is incidental to another aetiology. Stratification of smokers into less than or more than one pack a day also appears to have no scientific basis. The study is further confounded by inclusion of a relatively large proportion of patients for whom the amount of alcohol consumption and extent of smoking was unknown $(27 \%$ and $20 \%$, respectively).

It is well established that there is a close correlation between tobacco consumption and alcohol intake ${ }^{26}$ with heavy drinkers often being heavy smokers, and vice versa. ${ }^{28}{ }^{29}$ This high prevalence of smoking among alcoholics is a critical issue because unless stringent assessments of drinking and smoking are included in the study protocol, it is difficult to ascertain the relative pathogenic roles of the two factors in tissue injury. In order to conclusively delineate the role of smoking in the initiation or progression of alcohol induced injury, it is important to ensure that lifetime consumption of alcohol and tobacco is accurately measured, preferably in a prospective fashion.

Gut 2005;54:446-447.

doi: $10.1136 /$ gut.2004.048785 
Authors' affiliations

M V Apte, R C Pirola, J S Wilson, Pancreatic

Research Group, The University of New South

Wales, Sydney, Australia

Correspondence to: Professor J Wilson, South Western Sydney Clinical School, The University of New South Wales, Level 2, Thomas and Rachel Moore Education Centre, Liverpool Hospital, Liverpool, NSW 2170, Australia; js.wilson@unsw.edu.au

Conflict of interest: None declared.

\section{REFERENCES}

1 Dreiling DA, Koller M. The natural history of alcoholic pancreatitis: Update 1985. Mt Sinai J Med 1985;52:340-2.

2 Steinberg W, Tenner S. Acute pancreatitis. N Engl J Med 1994;330:1198-210.

3 Majumdar AP, Davis GA, Dubick MA, et al. Nicotine stimulation of protein secretion from isolated rat pancreatic acini. Am J Physiol 1985;248:G158-63.

4 Chowdhury P, Hosotani R, Chang L, et al. Metabolic and pathologic effects of nicotine on gastrointestinal tract and pancreas of rats. Pancreas 1990;5:222-9.

5 Balldin G, Borgstrom A, Eddeland A, et al. Elevated serum levels of pancreatic secretory proteins in cigarette smokers after secretin stimulation. J Clin Invest 1980;66:159-62.

6 Andriulli A, Masoero G, Amato A, et al. Serum immunoreactive cationic trypsinogen response to secretin in normal subjects. Am J Gastroenterol 1983;78:579-83.
7 Dubick MA, Conteas CN, Billy HT, et al. Raised serum concentrations of pancreatic enzymes in cigarette smokers. Gut 1987;28:330-5.

8 Bynum TE, Solomon TE, Johnson LR, et al. Inhibition of pancreatic secretion in man by cigarette smoking. Gut 1972;13:361-5.

9 Konturek SJ, Solomon TE, McCreight WG, et al. Effects of nicotine on gastrointestinal secretions. Gastroenterology 1971;60:1098-105.

10 Hartwig W, Werner J, Ryschich E, et al. Cigarette smoke enhances ethanol-induced pancreatic injury. Pancreas 2000;21:272-8.

11 Maisonneuve $P$, Lowenfels AB, Müllhaupt $B$, et al. Cigarette smoking accelerates progression of alcoholic chronic pancreatitis. Gut 2005;54:510-4.

12 Bourliere $M$, Barthet $M$, Berthezene $P$, et al. Is tobacco a risk factor for chronic pancreatitis and alcoholic cirrhosis? Gut 1991;32:1392-5.

13 Haber PS, Wilson JS, Pirola RC. Smoking and alcoholic pancreatitis. Pancreas 1993;8:568-72.

14 Haber $\mathbf{P}$, Wilson J, Apte $M$, et al. Individual susceptibility to alcoholic pancreatitis: Still an enigma. J Lab Clin Med 1995;125:305-12.

15 Talamini G, Bassi C, Falconi M, et al. Cigarette smoking: An independent risk factor in alcoholic pancreatitis. Pancreas 1996;12:131-7.

16 Wilson JS, Gossat D, Tait A, et al. Evidence for an inherited predisposition to alcoholic pancreatitis. A controlled HLA typing study. Dig Dis Sci 1984:29.727-30

17 Haber PS, Wilson JS, McGarity BH, et al. Alpha 1 antitrypsin phenotypes and alcoholic pancreatitis. Gut 1991;32:945-8.

18 Norton ID, Apte MV, Dixson H, et al. Cystic fibrosis genotypes and alcoholic pancreatitis. $J$ Gastroenterol Hepatol 1998;13:496-500.

19 El-Saaid M, Abdulrazeg EM, Alfirevic A, et al. TNF- $\alpha$ promoter region gene polymorphisms in patients with alcohol-induced chronic pancreatitis. Gastroenterology 2001;120:A32.
20 Frenzer A, Butler WJ, Norton ID, et al. Polymorphism in alcohol-metabolizing enzymes, glutathione S-transferases and apolipoprotein $\mathrm{E}$ and susceptibility to alcohol-induced cirrhosis and chronic pancreatitis. J Gastroenterol Hepatol 2002;17:177-82.

21 Bernardino AL, Guarita DR, Mott CB, et al. CFTR, PRSS 1 and SPINK 1 mutations in the development of pancreatitis in Brazilian patients. JOP 2003;4:169-77.

22 Perri F, Piepoli A, Stanziale P, et al. Mutation analysis of the cystic fibrosis transmembrane conductance regulator (CFTR) gene, the cationic trypsinogen (PRSS1) gene, and the serine protease inhibitor, Kazal type 1 (SPINK1) gene in patients with alcoholic chronic pancreatitis. Eur J Hum Genet 2003;11:687-92.

23 Whitcomb DC. Genetic predisposition to alcoholic chronic pancreatitis. Pancreas 2003;27:321-6.

24 Witt $\mathrm{H}$, Luck W, Becker $M$, et al. Mutation in the SPINK 1 trypsin inhibitor gene, alcohol use, and chronic pancreatitis. JAMA 2001:285:2716-17.

25 Schneider A, Pfutzer RH, Barmada MM, et al. SPINK1/PSTI gene mutations in patients with alcoholic chronic pancreatitis from North America. Gastroenterology 2002;122:A563.

26 Mohan D, Chopra A, Sethi H. The co-occurrence of tobacco and alcohol in general population of metropolis Delhi. Indian J Med Res 2002;116:150-4.

27 Room R. Smoking and drinking as complementary behaviours. Biomed Pharmacother 2004;58:111-15.

28 Bobo JK, Husten C. Sociocultural influences on smoking and drinking. Alcohol Res Health 2000;24:225-32.

29 Anthony JC, Echeagaray-Wagner F. Epidemiologic analysis of alcohol and tobacco use-patterns of co-occurring consumption and dependence in the United States. Alcohol Res Health 2000;24:201-8.

\section{Thalidomide and cancer cachexia: old problem, new hope?}

\section{Stroud}

\section{Thalidomide is safe and may be effective in attenuating severe weight loss in patients with advanced pancreatic cancer. This may also grant benefit in terms of improved physical function}

$\mathrm{T}$ he cancer cachexia syndrome is common. Significant weight loss occurs in approximately $50 \%$ of oncology patients, with even higher values in those with gastrointestinal tumours. ${ }^{1}$ In pancreatic cancer, approximately $80 \%$ of patients will become severely malnourished. The development of cachexia is not only distressing for patients and their families, it is also associated with a much worse clinical outcome. Malnourished patients undergoing surgery for cancer have morbidity and mortality rates of three to four times those of their better nourished counterparts, ${ }^{2}$ and wasted weakened patients also tolerate chemoradiation poorly. Ultimately, malnutrition itself can be considered to be the final cause of death in approximately $30 \%$ of cancer patients. It occurs once patients have lost about one third of their premorbid body weight.

Historical explanations for the causes of cancer induced wasting have been varied. Some experts have claimed that the dominant cause of weight loss is heightened energy demands, attributable to both the needs for tumour growth and tumour triggered changes in metabolism of tissues distant to the malignant process. However, although studies confirm that the resting metabolism of cancer patients is often elevated, ${ }^{3}$ total energy requirements are frequently lower than normal as patients who feel unwell do little physical activity. ${ }^{4}$ Furthermore, as cancer patients lose weight, their metabolic requirements fall further still. This type of argument has led other authorities to suggest that anorexia is the prime cause of wasting ${ }^{5}$ but once again, this cannot provide a full explanation. Although appetite loss and consequent falls in dietary intake can be profound (especially with gastrointestinal tumours), augmenting intakes using nutritional supplements is remarkably ineffective at slowing, let alone reversing, the wasting process. ${ }^{6}$ Furthermore, the pattern of tissue loss in cachexia is very different from that induced by starvation alone. Loss of lean tissues is more marked and it appears that cancers can trigger specific proteolytic and lipolytic pathways, as well as activate neuroendocrine systems that upregulate metabolism. ${ }^{78}$ Indeed, the changes in many ways are similar to those seen in the catabolic response to acute injury or sepsis.

Greater comprehension of the mechanisms underlying cancer cachexia has come from examination of the systems that regulate cellular metabolism. As 
with the acute phase response, it appears to be alterations in cytokines that lead to altered metabolic activity. Cancer patients have been shown to have elevated production of proinflammatory cytokines such as tumour necrosis factor $\alpha$, interleukin 1 , and interleukin 6, either produced by the tumour itself or released as part of a host response. ${ }^{9}$ These cytokines directly influence appetite, metabolic demands, and relative substrate utilisation, and indeed it appears that survival time in pancreatic cancer may relate to varying genetic propensity for proinflammatory cytokine production. ${ }^{10}$ In addition to changes in cytokines, cancer patients also have alterations in both leukotriene and prostaglandin-type eicosanoids, which can effect tissue loss through local changes in inflammatory status and systemic effects from upregulation of the acute phase response. ${ }^{11}$

Improved understanding of the triggers and responses underlying cancer cachexia offers new targets for potential therapeutic intervention. These are sorely needed as most attempts to date have met with very little success. Although perioperative nutritional support in malnourished cancer patients can improve perioperative outcome, ${ }^{12}$ the benefits appear to accrue from improved wound healing and greater resistance to infection rather than through weight gain per se; trials of nutritional supplements in cancer cachexia patients who are not undergoing surgery show very little benefit in terms of body weight, functional capacity, quality of life, or survival. ${ }^{6}$ Some interventions however have shown promise. These include megesterol acetate ${ }^{13}$ and downregulation of proinflammatory cytokine and eicosanoid pathways using fish oil supplements, ${ }^{14}$ although in the latter studies the benefits appeared to be confined to a subgroup of patients (perhaps those with an increased genetic propensity for inflammation). Furthermore, the benefits of megesterol acetate and fish oil supplementation seem confined to modest reductions in rates of body weight loss, with little change in either quality or length of remaining life.

In this issue of Gut, Gordon and colleagues ${ }^{15}$ report on the use of thalidomide for the cachexia of pancreatic cancer (see page 540). Thalidomide has a number of actions that make it a potentially useful anticancer agent. These include inhibition of angiogenesis, modulation of adhesion molecules, inhibition of cyclooxygenase 2, and stimulation of immune responses. ${ }^{16}$ Previous studies have confirmed significant thalidomide activity in multiple myeloma and some activity in myelofibrosis and myelodysplastic syndromes. Its activity in solid tumours however is less definite. ${ }^{17}$ Although thalidomide appears to have some potential in the treatment of Kaposi's sarcoma, malignant melanoma, renal carcinoma, and prostatic cancer, it has not proved effective in tumours of the head and neck, breast, or ovary. ${ }^{17}$ Nevertheless, the potential use of thalidomide in pancreatic cancer cachexia may relate to a completely different property of the drug which does not necessarily target the tumour itself. Thalidomide has quite powerful anti-tumour necrosis factor $\alpha$ effects which might alter the cytokine triggers of the wasting response. ${ }^{16}$

A small open label study of thalidomide in oesophageal cancer patients with weight loss was published last year, suggesting some attenuation of wasting. ${ }^{18}$ Now, Gordon and colleagues ${ }^{15}$ report a larger, double blind, randomised controlled trial of its use in advanced inoperable pancreatic cancer patients with more than a $10 \%$ decline in recent weight. This appears to show that this fairly cheap oral agent, thought to be reasonably safe if kept away from opportunities for causing birth defects, does ameliorate the wasting process and, furthermore, that maintaining better weight grants some benefit in terms of improved physical function.

Unfortunately, as with the studies of megesterol acetate and fish oil, the reduction in cancer related wasting reported in this new study of thalidomide had no definite effect on survival times. As death in many pancreatic cancer patients is frequently a direct result of malnutrition, this lack of benefit is a little surprising, and it should force consideration as to whether attenuating the cachectic process might result in disadvantages which offset any nutritional gains. From a teleological point of view, catabolism in general must grant survival advantage, perhaps through release of nutrients that are critical for normal immunological and acute phase responses. This may explain why measures which markedly reduce catabolism, such as growth hormone, appear to actually worsen rather than improve clinical outcome in severe injury or infection. ${ }^{19}$ However, although it is easy to see how catabolism might have evolved to grant survival advantage in injury and infection, the fact that most cancers affect individuals beyond their reproductive years and are fatal if left untreated makes it near impossible to imagine how natural selection could have led to the evolution of the cachexia response. Instead, it would seem more likely that it is an unfortunate "coincidence" of nature which, in turn, makes it reassuringly unlikely that turning off the cachectic response will have adverse effects on clinical outcome. Clearly, larger multicentre studies of thalidomide in pancreatic and other gastrointestinal cancers are required and should be undertaken as soon as possible.

Gut 2005;54:447-448.

doi: $10.1136 /$ gut.2004.053330

Correspondence to: $\operatorname{Dr} M$ Stroud, Institute of Human Nutrition, Mail Point 113, F Level, Centre Block, Southampton General Hospital, Tremona Rd, Southampton SO16 6YD, UK; m.a.stroud@soton.ac.uk

Conflict of interest: None declared.

\section{REFERENCES}

1 Palesty JA, Dudrick SJ. What we have learned about cachexia in gastrointestinal cancer. Dig Dis 2003;21:198-213.

2 Buzby GP, Mullen JP, Matthews DC. Prognostic nutritional index in gastrointestinal surgery. Am J Surg 1980;139:160.

3 Bosaeus I, Daneryd P, Svanberg E, et al. Dietary intake and resting energy expenditure in relation to weight loss in unselected cancer patients. Int J Cancer 2001;93:380-3.

4 Gibney $E$, Elia $M$, Jebb SA, et al. Total energy expenditure in patients with small-cell lung cancer: results of a validated study using the bicarbonate-urea method. Metabolism 1997;46:1412-17.

5 Jebb SA. Sir David Cuthbertson Medal Lecture. Energy metabolism in cancer and human immunodeficiency virus infection. Proc Nutr Soc 1997;56:763-75.

6 Barber MD, Ross JA, Fearon KC. Cance cachexia. Surg Oncol 1999:8:133-41.

7 Tisdale MJ. Cancer anorexia and cachexia. Nutrition 2001;17:438-42.

8 Wigmore SJ, Todorov PT, Barber MD, et al. Characteristics of patients with pancreatic cancer expressing a novel cancer cachectic factor. Br J Surg 2000;87:53-8.

9 Ramos EJ, Suzuki S, Marks D, et al. Cance anorexia-cachexia syndrome: cytokines and neuropeptides. Curr Opin Clin Nutr Metab Care 2004;7:427-34.

10 Barber MD, Powell JJ, Lynch SF, et al. A polymorphism of the interleukin-1 beta gene influences survival in pancreatic cancer. Br J Cancer 2000:83:1443-7.

11 Ross JA, Fearon KC. Eicosanoid-dependent cancer cachexia and wasting. Curr Opin Clin Nutr Metab Care 2002;5:241-8.

12 Stroud MA. Peri-operative nutrition. In: Johnson C, Taylor I, eds. Advances in Surgery. London: Royal Society of Medicine Press, 2002:37-46.

13 Pascual Lopez A, Roquei Figuls M, Urrutia Cuchi G, et al. Systematic review of megestrol acetate in the treatment of anorexia-cachexia syndrome. J Pain Symptom Manage 2004; 27:360-9

14 Burns CP, Halabi S, Clamon G, et al. Phase II study of high-dose fish oil capsules for patients with cancer-related cachexia. Cancer 2004; 101:370-8.

15 Gordon JN, Trebble TM, Ellis RD, et al. Thalidomide in the treatment of cancer cachexia: a randomised placebo controlled trial. Gut 2005;54:540-5

16 Fanelli M, Sarmiento R, Gattuso D, et al. Thalidomide: a new anticancer drug? Expert Opin Investig Drugs 2003;12:1211-25.

17 Eleutherakis-Papaiakovou V, Bamias A, Dimopoulos MA. Thalidomide in cancer medicine. Ann Oncol 2004; 15:1 151-60.

18 Khan ZH, Simpson EJ, Cole AT, et al. Oesophageal cancer and cachexia: the effect of short-term treatment with thalidomide on weight loss and lean body mass. Aliment Pharmacol Ther 2003; 17:677-82.

19 Ruokonen E, Takala J. Dangers of growth hormone therapy in critically ill patients. Ann Med 2000;32:317-22. 